\title{
Cascade-Based Controlled Attitude Synchronization and Tracking of Spacecraft in Leader-Follower Formation
}

\author{
Rune Schlanbusch, ${ }^{1}$ Antonio Loría, ${ }^{2}$ and Per Johan Nicklasson ${ }^{1}$ \\ ${ }^{1}$ Department of Technology, Narvik University College, PB 385, 8505 Narvik, Norway \\ ${ }^{2}$ CNRS, LSS-SUPELEC, 3 rue Joliot Curie, 91192 Gif-sur-Yvette, France
}

Correspondence should be addressed to Rune Schlanbusch, runsch@hin.no

Received 30 June 2010; Revised 5 January 2011; Accepted 23 February 2011

Academic Editor: Giampiero Campa

Copyright (c) 2011 Rune Schlanbusch et al. This is an open access article distributed under the Creative Commons Attribution License, which permits unrestricted use, distribution, and reproduction in any medium, provided the original work is properly cited.

\begin{abstract}
We propose controllers for leader-follower attitude synchronization of spacecraft formations in the presence of disturbances, that is, the leader spacecraft is controlled to follow a given reference, while a follower spacecraft is controlled to synchronize its motion with the leader's. In the ideal disturbance-free scenario, we show that synchronization takes place asymptotically. Moreover, we show the property of uniform practical asymptotic stability which implies that the synchronization is robust to bounded disturbances.
\end{abstract}

\section{Introduction}

In recent years, formation flying has become an increasingly popular subject of study. This is a new method of performing space operations, by replacing large and complex spacecraft with an array of simpler microspacecraft, bringing out new possibilities and opportunities of cost reduction, redundancy, and improved resolution aspects of onboard payload. One of the main challenges is the requirement of synchronization between spacecraft; robust and reliable control of relative position and attitude is necessary to make the spacecraft cooperate to gain the possible advantages made feasible by spacecraft formations. For fully autonomous spacecraft formations, both path- and attitude-planning must be performed online which introduces challenges like collision avoidance and restrictions on pointing instruments towards required targets, with the lowest possible fuel expenditure.

Synchronization of dynamical systems was first studied by Christian Huygens in the XVIIth century. In recent years, the problem has obtained increasing interest in various research areas due to its impact in technology development and challenges it imposes; see, for example, [1-4].

Model-based controlled synchronization consists in using the physics laws and control theory in order to induce synchronization in dynamical systems. Successful instances include synchronization of robot manipulators $[5,6]$, leader-follower spacecraft formations [7-10], and ship replenishment operations [11, 12]. Another form of synchronization is consensus, in which a group of systems coordinate their motion without any subsystem having a higher hierarchy with respect to the others. An instance of consensus is cooperative control in which a group of systems is controlled in a way that they collaborate in order to achieve a task as a team of agents. Examples may be found in the areas of autonomous vehicles [13$15]$, underactuated marine vessels $[16,17]$, and rigid bodies [18-20].

In this paper, we address the simultaneous control problems of attitude tracking and leader-follower synchronization. That is, we propose a tracking controller for the leader spacecraft which makes it follow a prescribed reference. Independently, we construct a synchronization control law for the follower spacecraft which makes it track the attitude of the leader, thereby synchronizing in the classical master-slave configuration.

Our controllers are reminiscent of classical tracking controllers for robot manipulators passivity-based control which exploits the system's physical properties; see [21]. For tracking control, see the passivity-based PD+ of [22] and the wrongly called "sliding-mode" controller of [23] which may rather be casted in the passivity-based framework. 


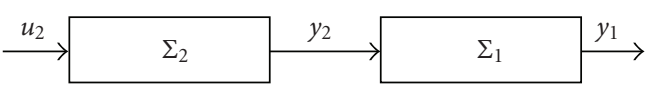

Figure 1: Cascade interconnection of two dynamical systems.

Although insightful, these popular control approaches for robot manipulators may not be directly applied in spacecraft tracking control and synchronization. The first obstacle is the specificity of spacecraft nonlinear models, expressed in quaternion coordinates. We revise the model in the following section.

Besides the difficulties imposed by the modeling of spacecraft, simultaneous tracking control and master-slave synchronization implicitly suggest controlling the leader spacecraft towards a reference independently of the slave system dynamics. Correspondingly, the synchronization controller inevitably couples the follower spacecraft to the dynamics of the leader. However, the synchronization controller is demanded to achieve the task regardless of the master dynamics as well as the reference that system intends to track. The ability to control two coupled systems separately is called separation principle and is known not to hold in general for nonlinear systems (see e.g. [24]). This is where cascades theory enters in play.

Cascaded systems theory consists in analyzing complex systems by dividing them in subsystems simpler to control and to analyze (see [25] and references within). It must be emphasized that such representation is purely schematic, for the purpose of analysis only. Generally speaking, the stability analysis problem consists in finding conditions for two systems as in Figure 1 so that, considering that both subsystems separately are stable, they conserve that property when interconnected.

In the context of the present paper, the block on the left corresponds to the leader system in closed loop with a tracking controller, while the block on the right consists in the follower spacecraft in closed loop with the synchronization controller. The blocks are interconnected via the tracking errors of the leader system. Hence, in the ideal case, when the leader spacecraft is perfectly controlled, the systems are decoupled.

The topic of cascaded systems have received a great deal of attention and has successfully been applied to a wide number of applications. In [26], a cascaded adaptive control scheme for marine vehicles including actuator dynamics was introduced, while [27] solved the problem of synchronization of two pendula through use of cascades. The authors of [28] studied the problem of global stabilizability of feedforward systems by a systematic recursive design procedure for autonomous systems, while time-varying systems were considered in [29] for stabilization of robust control, while [30] established sufficient conditions for uniform global asymptotical stability (UGAS) of cascaded nonlinear timevarying systems. The aspect of practical and semiglobal stability for nonlinear time-varying systems in cascade was pursued in $[31,32]$. A stability analysis of spacecraft formations including both leader and follower using relative coordinates is presented in [10], where the controller-observer scheme is proven input-to-state stable, and backstepping was applied in [17] for leader-follower formation control of multiple underactuated autonomous underwater vehicles (AUVs). For the control problems at hand, we show that the closed-loop system has the property of uniform asymptotic stability. Significantly, uniform asymptotic stability guarantees robustness with respect to bounded disturbances. In this regard, we extend our result to the case where bounded perturbations affect the system (atmospheric drag, gravity gradient, etc.). In this scenario, we guarantee uniform practical asymptotic stability. This pertains to the case when there exists a steady-state tracking and synchronization error which can be arbitrarily diminished via an appropriate tuning of the control parameters.

The contribution of this paper is application of the framework for stability analysis of cascaded systems of rigid bodies in leader-follower formation and synchronizvation of $\mathrm{PD}+$ and sliding surface control laws adapted for quaternion space. The equilibrium points of the $\mathrm{PD}+$ controller in closed loop with the rigid body dynamics are proven uniformly asymptotically stable (UAS) when disturbances are considered known, while a sliding surface controller is utilized to prove uniform practical asymptotical stability (UPAS) when disturbances are considered unknown but bounded. Simulation results of a leader-follower spacecraft formation are presented to show the performance of our proposed control laws.

The rest of the paper is organized as follows: in Section 2, we recall the modeling of rigid bodies in quaternion coordinates and present the main theoretical tools of cascaded systems theory, which are instrumental to our control design. Controller design is presented in Section 3 for known disturbances while for unknown but upper-bounded disturbances in Section 4. Simulation results are presented in Section 5, and everything is wrapped up with conclusions in Section 6.

\section{Mathematical Background}

In the following, we denote by $\dot{\mathbf{x}}$ the time derivative of a vector $\mathbf{x}$, that is, $\dot{\mathbf{x}}=d \mathbf{x} / d t$; moreover, $\ddot{\mathbf{x}}=d^{2} \mathbf{x} / d t^{2}$, and $\|\cdot\|$ denotes the $\ell^{2}$ norm of vectors and induced $\ell^{2}$ norm of matrices. Coordinate reference frames are denoted by $\mathcal{F}^{(\cdot)}$, where the superscript denotes the frame in question. Moreover, we denote a rotation matrix between frame $a$ and frame $b$ by $\mathbf{R}_{a}^{b} \in \mathrm{SO}(3)$, and the angular velocity of frame $a$ relative to frame $b$, referenced in frame $c$, is denoted by $\boldsymbol{\omega}_{b, a}^{c} \in \mathbb{R}^{3}$. We denote by $\mathbf{x}\left(t ; t_{0}, \mathbf{x}\left(t_{0}\right)\right)$ the solutions of the differential equation $\dot{\mathbf{x}}=f(t, \mathbf{x})$ with initial conditions $\left(t_{0}, \mathbf{x}\left(t_{0}\right)\right)$. When the context is sufficiently explicit, we may omit the arguments of a function, vector or matrix.

2.1. Cartesian Coordinate Frames. The coordinate reference frames used throughout the paper are defined as follows.

Earth-Centered Inertial Frame. The Earth-centered inertial (ECI) frame is denoted by $\mathcal{F}^{i}$ and has its origin in the center of the Earth. The axes are denoted by $x_{i}, y_{i}$, and $z_{i}$, where 
the $z_{i}$-axis is directed along the axis of rotation of the Earth toward the celestial North Pole, the $x_{i}$-axis is pointing in the direction of the vernal equinox, $\mathbf{Y}$, which is the vector pointing from the center of the sun toward the center of the Earth during the vernal equinox, and finally the $y_{i}$-axis completes the right-handed orthonormal frame.

Spacecraft Orbit Reference Frame. The orbit frame is denoted by $\mathcal{F}^{s o}$, where the sub-/superscript $s=l, f$ denotes the leader and follower spacecraft, respectively, such that we throughout the paper denote, for example, $\mathcal{F}^{l o}$ and $\mathcal{F}^{f o}$ as $\mathcal{F}^{s o}$, which has its origin located in the center of the mass of the spacecraft. The $\mathbf{e}_{r}$-axis in the frame coincides with the vector $\mathbf{r}=\left[r_{x}, r_{y}, r_{z}\right]^{\top}$ from the center of the Earth to the spacecraft, and the $\mathbf{e}_{h}$-axis is parallel to the orbital angular momentum vector, pointing in the orbit normal direction. The $\mathbf{e}_{\theta}$-axis completes the right-handed orthonormal frame. The basis vectors of the frame can be defined as

$$
\mathbf{e}_{r}=\frac{\mathbf{r}}{r}, \quad \mathbf{e}_{\theta}=\mathbf{e}_{h} \times \mathbf{e}_{r}, \quad \mathbf{e}_{h}=\frac{\mathbf{h}}{h},
$$

where $\mathbf{h}=\mathbf{r} \times \dot{\mathbf{r}}$ is the angular momentum vector of the orbit, $h=|\mathbf{h}|$ and $r=|\mathbf{r}|$. This frame is also known as the local vertical/local horizontal (LVLH) frame.

Spacecraft Body Reference Frame. The body frame of the spacecraft is denoted by $\mathcal{F}^{s b}$ and is located at the center of the mass of the spacecraft, and its basis vectors are aligned with the principle axis of inertia.

2.2. Quaternions. The attitude of a rigid body is often represented by a rotation matrix $\mathbf{R} \in \mathrm{SO}$ (3) fulfilling

$$
\operatorname{SO}(3)=\left\{\mathbf{R} \in \mathbb{R}^{3 \times 3}: \mathbf{R}^{\top} \mathbf{R}=\mathbf{I}, \operatorname{det} \mathbf{R}=1\right\},
$$

which is the special orthogonal group of order three. Quaternions are often used to parameterize members of $\mathrm{SO}(3)$ where the unit quaternion is defined as $\mathbf{q}=\left[\eta, \boldsymbol{\epsilon}^{\top}\right]^{\top} \in$ $\mathrm{S}^{3}=\left\{\mathbf{x} \in \mathbb{R}^{4}: \mathbf{x}^{\top} \mathbf{x}=1\right\}$, where $\eta \in \mathbb{R}$ is the scalar part and $\boldsymbol{\epsilon} \in \mathbb{R}^{3}$ is the vector part. The rotation matrix may be described by [33]

$$
\mathbf{R}=\mathbf{I}+2 \eta \mathbf{S}(\boldsymbol{\epsilon})+2 \mathbf{S}^{2}(\boldsymbol{\epsilon})
$$

where the matrix $\mathbf{S}(\cdot)$ is the cross product operator defined as

$$
\mathbf{S}(\boldsymbol{\epsilon})=\boldsymbol{\epsilon} \times=\left[\begin{array}{ccc}
0 & -\epsilon_{z} & \epsilon_{y} \\
\epsilon_{z} & 0 & -\epsilon_{x} \\
-\epsilon_{y} & \epsilon_{x} & 0
\end{array}\right]
$$

where $\boldsymbol{\epsilon}=\left[\epsilon_{x}, \epsilon_{y}, \epsilon_{z}\right]^{\top}$. The inverse rotation can be performed by using the inverse conjugate of $\mathbf{q}$ as $\overline{\mathbf{q}}=\left[\eta,-\boldsymbol{\epsilon}^{\top}\right]^{\top}$. The set $S^{3}$ forms a group with quaternion multiplication, which is distributive and associative, but not commutative, and the quaternion product of two arbitrary quaternions $\mathbf{q}_{1}$ and $\mathbf{q}_{2}$ is defined as [33]

$$
\mathbf{q}_{1} \otimes \mathbf{q}_{2}=\left[\begin{array}{c}
\eta_{1} \eta_{2}-\boldsymbol{\epsilon}_{1}^{\top} \boldsymbol{\epsilon}_{2} \\
\eta_{1} \boldsymbol{\epsilon}_{2}+\eta_{2} \boldsymbol{\epsilon}_{1}+\mathbf{S}\left(\boldsymbol{\epsilon}_{1}\right) \boldsymbol{\epsilon}_{2}
\end{array}\right] .
$$

2.3. Kinematics and Dynamics. The time derivative of (3) can be written as [33]

$$
\dot{\mathbf{R}}_{b}^{a}=\mathbf{S}\left(\boldsymbol{\omega}_{a, b}^{a}\right) \mathbf{R}_{b}^{a}=\mathbf{R}_{b}^{a} \mathbf{S}\left(\boldsymbol{\omega}_{a, b}^{b}\right)
$$

and the kinematic differential equations can be expressed as [33]

$$
\dot{\mathbf{q}}_{s}=\mathbf{T}\left(\mathbf{q}_{s}\right) \boldsymbol{\omega}_{i, s b}^{s b}
$$

where

$$
\mathbf{T}\left(\mathbf{q}_{s}\right)=\frac{1}{2}\left[\begin{array}{c}
-\boldsymbol{\epsilon}_{s}^{T} \\
\eta_{s} \mathbf{I}+\mathbf{S}\left(\boldsymbol{\epsilon}_{s}\right)
\end{array}\right] \in \mathbb{R}^{4 \times 3} .
$$

The dynamical model of a rigid body can be described by a differential equation for angular velocity and is deduced from Euler's moment equation. This equation describes the relationship between applied torque and angular momentum on a rigid body as [34]

$$
\mathbf{J}_{s} \dot{\boldsymbol{\omega}}_{i, s b}^{s b}=-\mathbf{S}\left(\boldsymbol{\omega}_{i, s b}^{s b}\right) \mathbf{J}_{s} \boldsymbol{\omega}_{i, s b}^{s b}+\boldsymbol{\tau}_{s}^{s b}
$$

where $\boldsymbol{\tau}_{s}^{s b} \in \mathbb{R}^{3}$ is the total torque working on the body frame and $\mathbf{J}_{s}=\operatorname{diag}\left\{J_{s x}, J_{s y}, J_{s z}\right\} \in \mathbb{R}^{3 \times 3}$ is the moment of inertia. The torque working on the body is expressed as $\boldsymbol{\tau}_{s}^{s b}=\boldsymbol{\tau}_{s a}^{s b}+$ $\boldsymbol{\tau}_{s d}^{s b}$, where $\boldsymbol{\tau}_{s d}^{s b}$ is the disturbance torque and $\boldsymbol{\tau}_{s a}^{s b}$ is the actuator (control) torque. It might be desirable to express the rotation between the body frame and the orbit frame which can be done by applying

$$
\boldsymbol{\omega}_{s, s b}^{s b}=\boldsymbol{\omega}_{i, s b}^{s b}-\mathbf{R}_{i}^{s b} \boldsymbol{\omega}_{i, s}^{i},
$$

where $\boldsymbol{\omega}_{i, s}^{i}=\mathbf{S}\left(\mathbf{r}_{s}^{i}\right) \mathbf{v}_{s}^{i} / \mathbf{r}_{s}^{i \top} \mathbf{r}_{s}^{i}$, and $\mathbf{r}_{s}^{i}$ and $\mathbf{v}_{s}^{i}$ are the spacecraft radius and velocity vector, respectively, represented in the inertial frame.

2.4. Cascaded Systems Theory. A typical nonlinear cascaded time-varying system on closed-loop dynamical form is expressed as

$$
\begin{gathered}
\Sigma_{1}: \dot{x}_{1}=f_{1}\left(t, x_{1}\right)+g(t, x) x_{2}, \\
\Sigma_{2}: \dot{x}_{2}=f_{2}\left(t, x_{2}\right),
\end{gathered}
$$

where $x_{1} \in \mathbb{R}^{n}, x_{2} \in \mathbb{R}^{m}, x=\left[x_{1}^{\top}, x_{2}^{\top}\right]^{\top}$, and the functions $f_{1}(\cdot, \cdot), f_{2}(\cdot, \cdot)$, and $g(\cdot, \cdot)$ are continuous in their arguments, locally Lipschitz in $x$, and uniform in $t$, and $f_{1}(\cdot, \cdot)$ is continuously differentiable in both arguments. Note that (12) is decoupled from (11), hence, it will be called the driving system, and its state enters as an input to the upper system with state $x_{1}$ through the interconnection term $g(t, x) x_{2}$.

In the context of this paper, the dynamics

$$
\dot{x}_{1}=f_{1}\left(t, x_{1}\right)
$$

represents the synchronization error dynamics of the leaderfollower configuration, assuming that perfect tracking is achieved for the leader system, that is, the tracking error for 
the latter is represented by $x_{2}$, and its closed-loop dynamics under tracking control will be represented by (12).

We will evoke [25, Theorem 1] to prove uniform asymptotic stability of the equilibrium point of a closedloop system on the form (11)-(12) under the following assumptions.

Assumption 1. There exist constants $c_{1}, c_{2}, \delta^{\prime}>0$ and a Lyapunov function $V\left(t, x_{1}\right)$ for (13) such that $V: \mathbb{R}_{\geq 0} \times$ $\mathbb{R}^{n} \rightarrow \mathbb{R}_{\geq 0}$ is positive definite, radially unbounded, $\dot{V}(t$, $\left.x_{1}\right) \leq 0$ and

$$
\begin{gathered}
\left\|\frac{\partial V}{\partial x_{1}}\right\|\left\|x_{1}\right\| \leq c_{1} V\left(t, x_{1}\right) \quad \forall\left\|x_{1}\right\| \geq \delta^{\prime}, \\
\left\|\frac{\partial V}{\partial x_{1}}\right\| \leq c_{2} \quad \forall\left\|x_{1}\right\| \leq \delta^{\prime} .
\end{gathered}
$$

Assumption 2. There exist two continuous functions $\xi_{1}, \xi_{2}$ : $\mathbb{R}_{\geq 0} \rightarrow \mathbb{R}_{\geq 0}$ such that $g(t, x)$ satisfies

$$
\|g(t, x)\| \leq \xi_{1}\left(\left\|x_{2}\right\|\right)+\xi_{2}\left(\left\|x_{2}\right\|\right)\left\|x_{1}\right\|
$$

Assumption 3. There exists a class $\mathcal{K}$ function $\alpha(\cdot)$ such that, for all $t_{0} \geq 0$, the trajectories of the system (12) satisfy

$$
\int_{t_{0}}^{\infty}\left\|x_{2}\left(t ; t_{0}, x_{2}\left(t_{0}\right)\right)\right\| d t \leq \alpha\left(\left\|x_{2}\left(t_{0}\right)\right\|\right) .
$$

The Theorem cited above may be extended to the case when the subsystems possess the weaker property of practical asymptotic stability. This pertains to the situation in which the errors do not converge to zero but to a bounded region that may be made arbitrarily small; see [35]. A related popular concept, for instance, in control of mechanical systems is that of ultimate boundedness. However, practical asymptotic stability is stronger than ultimate boundedness since the later is only a notion of convergence and does not imply stability in the sense of Lyapunov. In other words, the fact that the errors converge to a bounded region does not imply that they remain always arbitrarily close to it.

\section{Uniform Asymptotic Stabilization}

We are ready to present the first results on tracking and synchronization cascade-based control. The control strategy relies on using models for two single spacecraft coupled through synchronized control, and stability properties are proved using cascade theory for known disturbances, that is, we assume that the disturbances $\boldsymbol{\tau}_{l d}^{l b}$ and $\tau_{f d}^{f b}$ can be modeled correctly (see, e.g., $[34,36,37])$.

3.1. Problem Formulation. The control problem is to design two controllers; the first one makes the states $\mathbf{q}_{l}(t), \boldsymbol{\omega}_{i, l b}^{l b}(t)$, and $\dot{\omega}_{i, l b}^{l b}(t)$ converge towards the generated references specified as $\mathbf{q}_{d}(t), \boldsymbol{\omega}_{i, d}^{l b}(t)$, and $\dot{\boldsymbol{\omega}}_{i, d}^{l b}(t)$, satisfying the kinematic equation

$$
\dot{\mathbf{q}}_{d}=\mathbf{T}\left(\mathbf{q}_{d}\right) \boldsymbol{\omega}_{i, d}^{l b}
$$

and acts as a solution to the dynamical model presented in (9). It should be noted that we apply (10) and its derivative to the generated reference rather than the dynamical equation to keep a simple control law structure compared to [38]. The second controller is designed as a synchronizing controller to make the states $\mathbf{q}_{f}(t)$ and $\boldsymbol{\omega}_{i, f b}^{f b}(t)$ converge towards $\mathbf{q}_{l}(t)$ and $\boldsymbol{\omega}_{i, l b}^{f b}(t)$. The error quaternion $\tilde{\mathbf{q}}_{s}=\left[\tilde{\eta}_{s}, \tilde{\boldsymbol{\epsilon}}_{s}^{\top}\right]^{\top}$ is found by applying the quaternion product

$$
\tilde{\mathbf{q}}_{s}=\mathbf{q}_{s} \otimes \overline{\mathbf{q}}_{d}=\left[\begin{array}{c}
\eta_{s} \eta_{d}+\boldsymbol{\epsilon}_{s}^{\top} \boldsymbol{\epsilon}_{d} \\
\eta_{d} \boldsymbol{\epsilon}_{s}-\eta_{s} \boldsymbol{\epsilon}_{d}-\mathbf{S}\left(\boldsymbol{\epsilon}_{s}\right) \boldsymbol{\epsilon}_{d}
\end{array}\right],
$$

where the sub-/superscript $s=l, f$ denotes the leader and follower spacecraft, respectively, and the error kinematics can be expressed as [39]

$$
\dot{\tilde{\mathbf{q}}}_{s}=\frac{1}{2} \mathbf{T}\left(\widetilde{\mathbf{q}}_{s}\right) \mathbf{e}_{s \omega}
$$

where $\mathbf{e}_{s \omega}=\boldsymbol{\omega}_{i, s b}^{s b}-\boldsymbol{\omega}_{i, d}^{s b}$ is the angular velocity error. Due to the redundancy in the quaternion representation, $\tilde{\mathbf{q}}_{s}$ and $-\widetilde{\mathbf{q}}_{s}$ represent the same physical attitude, but, mathematically, it differs by a $2 \pi$ rotation about an arbitrary axis. Therefore, we are not able to achieve a global representation since the term global refers to the whole state space $\mathbb{R}^{n}$ according to [35]. Since both equilibrium points represent the same physical representation, the equilibrium point which requires the shortest rotation is usually chosen, thus minimizing the path length; hence, $\tilde{\mathbf{q}}_{s+}=\left[1, \mathbf{0}^{\top}\right]^{\top}$ is chosen if $\tilde{\eta}_{s}\left(t_{0}\right) \geq 0$ and $\widetilde{\mathbf{q}}_{s-}=$ $\left[-1, \mathbf{0}^{\top}\right]^{\top}$ is chosen if $\tilde{\eta}_{s}\left(t_{0}\right)<0$. For the positive equilibrium point, an attitude error function is chosen as $\mathbf{e}_{s q_{+}}=[1-$ $\left.\tilde{\eta}_{s}, \tilde{\boldsymbol{\epsilon}}_{s}^{\top}\right]^{\top}$ (see [9]), while, for the negative equilibrium point, the attitude error function is chosen as $\mathbf{e}_{s q_{-}}=\left[1+\tilde{\eta}_{s}, \widetilde{\boldsymbol{\epsilon}}_{s}^{\top}\right]^{\top}$. In accordance with general kinematic relations

$$
\dot{\mathbf{e}}_{s q \pm}=\mathbf{T}_{s e}\left(\mathbf{e}_{s q \pm}\right) \mathbf{e}_{s \omega}
$$

where

$$
\mathbf{T}_{s e}\left(\mathbf{e}_{s q \pm}\right)=\frac{1}{2}\left[\begin{array}{c} 
\pm \tilde{\boldsymbol{\epsilon}}_{s}^{\top} \\
\tilde{\eta}_{s} \mathbf{I}+\mathbf{S}\left(\tilde{\boldsymbol{\epsilon}}_{s}\right)
\end{array}\right]
$$

The attitude error function is chosen a priori and kept throughout the maneuver even if $\tilde{\eta}_{s}(t)$ should happen to switch sign for some $t$. The control problem is presented as a cascaded system on the form (11)-(12), where the states are defined as $\mathbf{x}_{1}=\left[\mathbf{e}_{f q}^{\top}, \mathbf{e}_{f \omega}^{\top}\right]^{\top}$ and $\mathbf{x}_{2}=\left[\mathbf{e}_{l q}^{\top}, \mathbf{e}_{l \omega}^{\top}\right]^{\top}$. The control objective is to stabilize the equilibrium point $\left(\mathbf{e}_{f q}(t), \mathbf{e}_{f \omega}(t), \mathbf{e}_{l q}(t), \mathbf{e}_{l \omega}(t)\right)=(\mathbf{0}, \mathbf{0}, \mathbf{0}, \mathbf{0})$ as $t \rightarrow \infty$ for all initial values.

3.2. Control of Leader Spacecraft. For control of the leader spacecraft attitude, we incorporate a model-dependent control law as in [40]. It is assumed that we have available information of its attitude $\mathbf{q}_{l}$, angular velocity $\boldsymbol{\omega}_{i, l b}^{l b}$, and inertia matrix $\mathbf{J}_{l}$, and, temporarily, we assume to know external perturbations. We choose the equilibrium such that $\mathbf{e}_{l q \pm}=\left[1 \mp \tilde{\eta}_{l}, \widetilde{\boldsymbol{\epsilon}}_{l}^{\top}\right]^{\top}$ is either the positive or negative 
equilibrium point, which does not change during the maneuver. By pure conventionalism, consider the positive equilibrium, that is, $\mathbf{e}_{l_{q}}=\mathbf{e}_{l_{q+}}$. We define desired attitude $\mathbf{q}_{d}(t)$, desired angular velocity $\boldsymbol{\omega}_{i, d}^{l b}(t)$, and desired angular acceleration $\dot{\boldsymbol{\omega}}_{i, d}^{l b}(t)$ which are all bounded functions. The control law is expressed as

$$
\boldsymbol{\tau}_{l a}^{l b}=\mathbf{J}_{l} \dot{\boldsymbol{\omega}}_{i, d}^{l b}-\mathbf{S}\left(\mathbf{J}_{l} \boldsymbol{\omega}_{i, l b}^{l b}\right) \boldsymbol{\omega}_{i, d}^{l b}-k_{l q} \mathbf{T}_{l e}^{\top} \mathbf{e}_{l q}-k_{l \omega} \mathbf{e}_{l \omega}-\boldsymbol{\tau}_{l d}^{l b},
$$

where $k_{l q}>0$ and $k_{l \omega}>0$ are feedback gain scalars. By insertion of (23) into (9), the system is on form (12), and, by applying the property $\mathbf{S}\left(\boldsymbol{\omega}_{i, l b}^{l b}\right) \mathbf{J}_{l} \boldsymbol{\omega}_{i, l b}^{l b}=-\mathbf{S}\left(\mathbf{J}_{l} \boldsymbol{\omega}_{i, l b}^{l b}\right) \boldsymbol{\omega}_{i, l b}^{l b}$, we obtain the closed-loop dynamics

$$
\mathbf{J}_{l} \dot{\mathbf{e}}_{l \omega}+\left(k_{l \omega} \mathbf{I}-\mathbf{S}\left(\mathbf{J}_{l} \boldsymbol{\omega}_{i, l b}^{l b}\right)\right) \mathbf{e}_{l \omega}+k_{l q} \mathbf{T}_{l e}^{\top} \mathbf{e}_{l q}=\mathbf{0} .
$$

A radial unbounded, positive definite Lyapunov function candidate is defined as

$$
V_{l}=\frac{1}{2} \mathbf{e}_{l \omega}^{\top} \mathbf{J}_{l} \mathbf{e}_{l \omega}+\frac{1}{2} \mathbf{e}_{l q}^{\top} k_{l q} \mathbf{e}_{l q}>0 \quad \forall \mathbf{e}_{l \omega} \neq \mathbf{0}, \mathbf{e}_{l q} \neq \mathbf{0} .
$$

Indeed, we have

$$
\frac{1}{2} \min \left\{j_{l m}, k_{l q}\right\}\left\|\mathbf{x}_{2}\right\|^{2} \leq V_{l} \leq \frac{1}{2} \max \left\{j_{l M}, k_{l q}\right\}\left\|\mathbf{x}_{2}\right\|^{2},
$$

where $j_{l m} \leq\left\|J_{l}\right\| \leq j_{l M}$. By differentiation of (25) and inserting (24) and (21), we obtain

$$
\dot{V}_{l}=\mathbf{e}_{l q}^{\top} k_{l q} \mathbf{T}_{l e} \mathbf{e}_{l \omega}+\mathbf{e}_{l \omega}^{\top}\left[\mathbf{S}\left(\mathbf{J}_{l} \boldsymbol{\omega}_{i, l b}^{l b}\right)-k_{l \omega} \mathbf{I}\right] \mathbf{e}_{l \omega}-\mathbf{e}_{l \omega}^{\top} k_{l q} \mathbf{T}_{l e}^{\top} \mathbf{e}_{l q},
$$

where the first part of the second term in (27) is zero because $\mathbf{S}\left(\mathbf{J}_{l} \boldsymbol{\omega}_{i, l b}^{l b}\right)$ is a skew-symmetric matrix, which leads to

$$
\dot{V}_{l}=-\mathbf{e}_{l \omega}^{\top} k_{l \omega} \mathbf{e}_{l \omega} \leq 0
$$

so the origin of the system is uniformly stable and the trajectories are bounded. The rest of the proof consists in showing that the position errors and the velocity tracking errors are square integrable. Then it suffices to invoke [41, Lemma 3].

Let $\mathcal{V}_{l}(t)=V_{l}\left(\mathbf{e}_{l q}(t), \mathbf{e}_{l \omega}(t)\right)$ and $\mathbf{x}_{2}(t)=\left[\mathbf{e}_{l q}(t)^{\top}\right.$, $\left.\mathbf{e}_{l \omega}(t)^{\top}\right]^{\top}$. Then, from (28), we obtain by integrating on both sides

$$
\begin{gathered}
\int_{t_{0}}^{t} \dot{\mathcal{V}}_{l}(s) d s=-\int_{t_{0}}^{t} \mathbf{e}_{l \omega}^{\top}(s) k_{l \omega} \mathbf{e}_{l \omega}(s) d s, \\
-\mathcal{V}_{l}(t)+\mathcal{V}_{l}\left(t_{0}\right)=k_{l \omega} \int_{t_{0}}^{t}\left\|\mathbf{e}_{l \omega}(s)\right\|^{2} d s .
\end{gathered}
$$

Since $-\mathcal{V}_{l}(t) \leq 0$, we can write

$$
\begin{aligned}
k_{l \omega} \int_{t_{0}}^{t}\left\|\mathbf{e}_{l \omega}\right\|^{2} d s & \leq \mathcal{V}_{l}\left(t_{0}\right) \\
& =\frac{1}{2}\left(\mathbf{e}_{l \omega}^{\top}\left(t_{0}\right) \mathbf{J}_{l} \mathbf{e}_{l \omega}\left(t_{0}\right)+\mathbf{e}_{l q}^{\top}\left(t_{0}\right) k_{l q} \mathbf{e}_{l q}\left(t_{0}\right)\right) \\
& \leq \frac{1}{2} \max \left\{j_{l M}, k_{l q}\right\}\left\|\mathbf{x}_{2}\left(t_{0}\right)\right\|^{2},
\end{aligned}
$$

and thus

$$
\int_{t_{0}}^{t}\left\|\mathbf{e}_{l \omega}(s)\right\|^{2} d s \leq c_{3}\left\|\mathbf{x}_{2}\left(t_{0}\right)\right\|^{2},
$$

where $c_{3}=\max \left\{j_{l M}, k_{l q}\right\} / 2 k_{l \omega}$.

Now let $W_{l}(t)=W_{l}\left(\mathbf{e}_{l q}(t), \mathbf{e}_{l \omega}(t)\right)$ such that

$$
\mathcal{W}_{l}(t)=\mathbf{e}_{l q}(t)^{\top} \mathbf{T}_{l e}(t) k_{l q} \mathbf{J}_{l} \mathbf{e}_{l \omega}(t),
$$

and, by differentiation, we obtain

$$
\begin{aligned}
\dot{W}_{l}(t)= & \dot{\mathbf{e}}_{l q}^{\top}(t) \mathbf{T}_{l e}(t) k_{l q} \mathbf{J}_{l} \mathbf{e}_{l \omega}(t) \\
& +\mathbf{e}_{l q}^{\top}(t) \dot{\mathbf{T}}_{l e}(t) k_{l q} \mathbf{J}_{l} \mathbf{e}_{l \omega}(t)+\mathbf{e}_{l q}^{\top}(t) \mathbf{T}_{l e}(t) k_{l q} \mathbf{J}_{l} \dot{\mathbf{e}}_{l \omega}(t) .
\end{aligned}
$$

By inserting $\dot{\mathbf{T}}_{l e}^{\top}(t) \mathbf{e}_{l q}(t)=\mathbf{G}_{l}(t) \mathbf{e}_{l \omega}(t)$, where

$$
\mathbf{G}_{l}(t)=\frac{1}{2}\left[\tilde{\eta}_{l}(t) \mathbf{I}+\mathbf{S}\left(\tilde{\boldsymbol{\epsilon}}_{l}(t)\right)\right]-\frac{1}{4} \mathbf{I},
$$

and (21) and (24) into (34), we obtain

$$
\begin{aligned}
\dot{\boldsymbol{W}}_{l}= & \mathbf{e}_{l \omega}^{\top}(t) \mathbf{T}_{l e}^{\top}(t) \mathbf{T}_{l e}(t) k_{l q} \mathbf{J}_{l} \mathbf{e}_{l \omega}(t)+\mathbf{e}_{l \omega}^{\top} \mathbf{G}_{l}^{\top}(t) k_{l q} \mathbf{J}_{l} \mathbf{e}_{l \omega}(t) \\
& -\mathbf{e}_{l q}^{\top}(t) \mathbf{T}_{l e}(t) k_{l q}\left[-\mathbf{S}\left(\mathbf{J}_{l} \boldsymbol{\omega}_{i, l b}^{l b}(t)\right)+k_{l \omega} \mathbf{I}\right] \mathbf{e}_{l \omega}(t) \\
& -\mathbf{e}_{l q}^{\top}(t) \mathbf{T}_{l e}(t) k_{l q}^{2} \mathbf{T}_{l e}^{\top}(t) \mathbf{e}_{l q}(t) \\
\leq & -c_{4}\left\|\mathbf{e}_{l q}\right\|^{2}+c_{5}\left\|\mathbf{e}_{l \omega}\right\|^{2}+\left\|\mathbf{e}_{l q}\right\| c_{6}\left\|\mathbf{e}_{l \omega}\right\|,
\end{aligned}
$$

where $c_{4}=k_{l q}^{2} / 4, c_{5}=k_{l q} j_{l M}\left\|\mathbf{G}_{l}^{\top}(t)\right\|$,

$$
c_{6}=\frac{k_{l q}}{2\left(\left\|\mathbf{S}\left(\mathbf{J}_{l} \boldsymbol{\omega}_{i, l b}^{l b}(t)\right)\right\|+k_{l \omega}\right)},
$$

and $\left\|\mathbf{G}_{l}^{\top}(t)\right\| \leq 3 / 4$. The last term of (36) can be rewritten as

$$
\left\|\mathbf{e}_{l q}\right\| c_{6}\left\|\mathbf{e}_{l \omega}\right\| \leq \kappa\left\|\mathbf{e}_{l \omega}(t)\right\|^{2}+\frac{c_{6}^{2}}{\kappa}\left\|\mathbf{e}_{l q}(t)\right\|^{2},
$$

and by choosing $\kappa \gg 1$ such that $c_{4}>2 c_{6}^{2} / \kappa$, we obtain

$$
\dot{\boldsymbol{W}}_{l}(t) \leq-\frac{c_{4}}{2\left\|\mathbf{e}_{l q}(t)\right\|^{2}+\left(c_{5}+\kappa\right)\left\|\mathbf{e}_{l \omega}(t)\right\|^{2}} .
$$

By applying the same line of arguments as in (29)-(31) and inequality (32), (39) may be expressed as

$$
\mathfrak{W}_{l}\left(t_{0}\right)+\left(c_{5}+\kappa\right) c_{3}\left\|\mathbf{x}_{2}\left(t_{0}\right)\right\|^{2} \geq \frac{c_{4}}{2} \int_{t_{0}}^{t}\left\|\mathbf{e}_{l q}(s)\right\|^{2} d s .
$$

By inserting the upper bound

$$
\begin{aligned}
\left\|\boldsymbol{W}_{l}\left(t_{0}\right)\right\| & \leq \frac{1}{2} \max \left\{k_{l q}, j_{l M}\right\}\left\|\mathbf{e}_{l q}\left(t_{0}\right)\right\|\left\|\mathbf{e}_{l \omega}\left(t_{0}\right)\right\| \\
& \leq \max \left\{k_{l q}, j_{l M}\right\}\left(\left\|\mathbf{e}_{l q}\left(t_{0}\right)\right\|^{2}+\left\|\mathbf{e}_{l \omega}\left(t_{0}\right)\right\|^{2}\right) \\
& \leq \max \left\{k_{l q}, j_{l M}\right\}\left\|\mathbf{x}_{2}\left(t_{0}\right)\right\|^{2}
\end{aligned}
$$


into (40), the expression may be written as

$$
\int_{t_{0}}^{t}\left\|\mathbf{e}_{l q}(s)\right\|^{2} d s \leq c_{7}\left\|\mathbf{x}_{2}\left(t_{0}\right)\right\|^{2},
$$

where $c_{7}=2\left(1 / 2 \max \left\{k_{l q}, j_{l M}\right\}+\left(c_{5}+\kappa\right) c_{3}\right) / c_{4}$.

We conclude from Lemma 3 of [41] that the origin is uniformly exponentially stable.

3.3. Control of Follower Spacecraft. For control of the follower spacecraft attitude, we incorporate a similar control law as in Section 3.2 but add terms for synchronization. For the control law, it is assumed that we have available information of the attitude and angular velocity for both spacecraft and inertia matrix, $\mathbf{J}_{f}$, and that all perturbations are known and accounted for. In the following, it is assumed that the equilibrium point is chosen such that $\mathbf{e}_{f q \pm}=\left[1 \mp \tilde{\eta}_{f}, \tilde{\boldsymbol{\epsilon}}_{f}^{\top}\right]^{\top}$ is either the positive or negative equilibrium point and does not change during the maneuver. The control law is expressed as

$$
\begin{aligned}
\boldsymbol{\tau}_{f a}^{f b}= & \mathbf{J}_{f} \dot{\boldsymbol{\omega}}_{i, d}^{f b}-\mathbf{S}\left(\mathbf{J}_{f} \boldsymbol{\omega}_{i, f b}^{f b}\right) \boldsymbol{\omega}_{i, d}^{f b} \\
& -k_{f q}\left(\mathbf{T}_{f e}^{\top} \mathbf{e}_{f q}-\mathbf{T}_{l e}^{\top} \mathbf{e}_{l q}\right)-k_{f \omega}\left(\mathbf{e}_{f \omega}-\mathbf{e}_{l \omega}\right)-\boldsymbol{\tau}_{f d}^{f b},
\end{aligned}
$$

where $k_{f q}>0$ and $k_{f \omega}>0$ are feedback gain scalars and the last two terms are for synchronization. By insertion of (43) into (9), we obtain the closed-loop rotational dynamics

$$
\begin{aligned}
\dot{\mathbf{e}}_{f \omega}=\mathbf{J}_{f}^{-1}[- & \left(k_{f \omega}-\mathbf{S}\left(\mathbf{J}_{f} \boldsymbol{\omega}_{i, f b}^{f b}\right)\right) \mathbf{e}_{f \omega} \\
& \left.-k_{f q} \mathbf{T}_{f e}^{\top} \mathbf{e}_{f q}+k_{f q} \mathbf{T}_{l e}^{\top} \mathbf{e}_{l q}+k_{f \omega} \mathbf{e}_{l \omega}\right] \\
= & f\left(\mathbf{x}_{1}\right)+g(\mathbf{x}) \mathbf{x}_{2},
\end{aligned}
$$

where $f\left(\mathbf{x}_{1}\right)$ is similar to the closed-loop system derived in Section 3.2 hence the proof of uniform asymptotic stability follows along similar lines.

The interconnection function is

$$
g(\mathbf{x})=\left[\begin{array}{cc}
\mathbf{J}_{f}^{-1} k_{f q} \mathbf{T}_{l e}^{\top} & \mathbf{0} \\
\mathbf{0} & \mathbf{J}_{f}^{-1} k_{f \omega}
\end{array}\right] .
$$

In what follows we show that Assumptions 1-3 hold and, hence, that the origin of the closed-loop system is uniformly asymptotically stable.

Proof of Assumption 1. We start by considering $\delta^{\prime}=1$ (where in this case $\delta^{\prime}$ is the parameter given in Assumption 1). By evaluating (14) on $V_{f}$, we obtain

$$
\begin{aligned}
\left\|\mathbf{e}_{f \omega}^{\top} \mathbf{J}_{f}+\mathbf{e}_{f q}^{\top} k_{f q}\right\|\left\|\mathbf{x}_{1}\right\| & \leq c_{1} V_{f}\left(\mathbf{x}_{1}\right) \\
& =\frac{c_{1}}{2}\left[\mathbf{e}_{f \omega}^{\top} \mathbf{J}_{f} \mathbf{e}_{f \omega}+\mathbf{e}_{f q}^{\top} k_{f q} \mathbf{e}_{f q}\right],
\end{aligned}
$$

and by applying the triangular inequality on the left side of (46) and squaring, we obtain

$$
\begin{aligned}
& \left(\left\|\mathbf{e}_{f \omega}^{\top} \mathbf{J}_{f}\right\|+\left\|\mathbf{e}_{f q}^{\top} k_{f q}\right\|\right)^{2}\left\|\mathbf{x}_{1}\right\|^{2} \\
& \quad \leq \frac{c_{1}^{2}}{4}\left[\mathbf{e}_{f \omega}^{\top} \mathbf{J}_{f} \mathbf{e}_{f \omega}+\mathbf{e}_{f q}^{\top} k_{f q} \mathbf{e}_{f q}\right]^{2} .
\end{aligned}
$$

On the left side of (47), we apply $\|x\|^{2}+\|y\|^{2}+2\|x\|\|y\| \leq$ $2\left(\|x\|^{2}+\|y\|^{2}\right)$ and $j_{f m} \leq\left\|\mathbf{J}_{f}\right\| \leq j_{f M}$ such that

$$
\begin{aligned}
& \left(\left\|\mathbf{e}_{f \omega}^{\top} \mathbf{J}_{f}\right\|+\left\|\mathbf{e}_{f q}^{\top} k_{f q}\right\|\right)^{2} \\
& \quad \leq 2 \varphi\left(\mathbf{e}_{f \omega}^{\top} \mathbf{J}_{f} \mathbf{e}_{f \omega}+\mathbf{e}_{f q}^{\top} k_{f q} \mathbf{e}_{f q}\right),
\end{aligned}
$$

where $\varphi=\max \left\{j_{f M}, k_{f q}\right\}$. By insertion of (48) in (47), we obtain

$$
2 \varphi\left(\mathbf{e}_{f \omega}^{\top} \mathbf{e}_{f \omega}+\mathbf{e}_{f q}^{\top} \mathbf{e}_{f q}\right) \leq \frac{c_{1}^{2} \rho}{4}\left(\mathbf{e}_{f \omega}^{\top} \mathbf{e}_{f \omega}+\mathbf{e}_{f q}^{\top} \mathbf{e}_{f q}\right),
$$

where $\rho=\min \left\{j_{f m}, k_{f q}\right\}$, and thus, we have to choose

$$
c_{1} \geq \sqrt{\frac{8 \varphi}{\rho}}
$$

to fulfill (14). For (15), we have that

$$
\left\|\mathbf{e}_{f \omega}^{\top} \mathbf{J}_{f}+\mathbf{e}_{f q}^{\top} k_{f q}\right\| \leq c_{2},
$$

and by using the triangular inequality and squaring and applying (48) we obtain

$$
2 \varphi^{2}\left\|\mathbf{x}_{1}\right\|^{2} \leq c_{2}^{2}
$$

Since $\left\|\mathbf{x}_{1}\right\| \leq 1$, we have to choose

$$
c_{2} \geq \sqrt{2} \varphi
$$

to fulfill (15), and thus Assumption 1 is fulfilled.

Proof of Assumption 2. Since $\left\|\mathbf{T}_{l e}\right\|=1 / 2$, (45) obviously fulfills the growth rate criteria of (16), such as

$$
\begin{aligned}
\|g(\mathbf{x})\| & \leq \frac{1}{2}\left[\left(\frac{1}{J_{x}^{2}}+\frac{1}{J_{y}^{2}}+\frac{1}{J_{z}^{2}}\right)\left(k_{f q}^{2}+4 k_{f \omega}^{2}\right)\right]^{1 / 2} \\
& =\xi_{1},
\end{aligned}
$$

where $\xi_{1}$ is constant, and thus Assumption 2 is fulfilled.

Proof of Assumption 3. This assumption holds because $\left\|\mathbf{x}_{2}(t)\right\|$ converges to zero exponentially fast.

We conclude that the equilibrium point $\left(\mathbf{e}_{q q}, \mathbf{e}_{l \omega}, \mathbf{e}_{f q}\right.$, $\left.\mathbf{e}_{f \omega}\right)=(\mathbf{0}, \mathbf{0}, \mathbf{0}, \mathbf{0})$ of the cascaded system is UAS.

The proof for the other equilibria follows mutatis mutandis.

\section{Robustness to Disturbances}

In this section, we develop our results from Section 3 by introducing unknown bounded disturbances. We use a control law reminiscent of the Slotine and Li controller for manipulators; see [23], based on a control structure which has often been shown to be favorable from a stability analysis point of view. In the case of bounded additive nonvanishing disturbances, a steady-state error is unavoidable; hence, only practical asymptotic stability may be obtained. Although the control approach is the same, the technical analysis tools are more sophisticated. We refer the reader to [32]. 
4.1. Control of Leader Spacecraft. We start by assuming that we have available controller gains according to $\theta_{1}=$ $\left[k_{f q}, k_{f \omega}\right], \theta_{2}=\left[k_{l q}, k_{l \omega}\right] \in \Theta_{1}=\Theta_{2}=\mathbb{R}_{>0}^{2}$. The uniform asymptotic stability in Section 3.2 is a result of the assumption that $\boldsymbol{\tau}_{l d}^{l b}$ is known and accounted for in the control law. We relax this assumption and assume that $\boldsymbol{\tau}_{l d}^{l b}$ is unknown, but bounded, and thus there exists a $\beta_{l}>0$ such that $\left\|\boldsymbol{\tau}_{l d}^{l b}\right\| \leq \beta_{l}$. Note that many of the disturbances for Earth-orbiting spacecraft can be reasonably well modeled as $\hat{\mathbf{f}}_{\mathrm{sd}}^{\mathrm{sb}}$, which can be added to the overall analysis such that $\tilde{\mathbf{f}}_{\mathrm{sd}}^{\mathrm{sb}}=\mathbf{f}_{\mathrm{sd}}^{s b}-\hat{\mathbf{f}}_{\mathrm{sd}}^{s b}$. This strategy can reduce the upper bound such that $\left\|\mathbf{f}_{\text {sd }}^{\tilde{s} b}(t)\right\|<\beta_{\widetilde{s}}<\beta_{s}$, based on the quality of the disturbance modeling. We apply the control law

$$
\begin{gathered}
\boldsymbol{\tau}_{l a}^{l b}=\mathbf{J}_{l} \dot{\boldsymbol{\omega}}_{i, r}^{l b}-\mathbf{S}\left(\mathbf{J}_{l} \boldsymbol{\omega}_{i, l b}^{l b}\right) \boldsymbol{\omega}_{i, r}^{l b}-k_{l q} \mathbf{T}_{l e}^{\top} \mathbf{e}_{l q}-k_{l \omega} \mathbf{s}_{l}, \\
\boldsymbol{\omega}_{i, r}^{l b}=\boldsymbol{\omega}_{i, d}^{l b}-\boldsymbol{\Gamma}_{l} \mathbf{T}_{l e}^{\top}\left(\mathbf{e}_{l q}\right) \mathbf{e}_{l q}, \\
\mathbf{s}_{l}=\boldsymbol{\omega}_{i, l b}^{l b}-\boldsymbol{\omega}_{i, r}^{l b}=\mathbf{e}_{l \omega}+\Gamma_{l} \mathbf{T}_{l e}^{\top} \mathbf{e}_{q l},
\end{gathered}
$$

where $k_{l q}>0, k_{l \omega}>0$ and $\boldsymbol{\Gamma}_{l}=\boldsymbol{\Gamma}_{l}^{\top}>0$ are feedback gains, and, by inserting (55) into (9), we obtain the closed-loop dynamics

$$
\dot{\mathbf{s}}_{l}=\mathbf{J}_{l}^{-1}\left[\mathbf{S}\left(\mathbf{J}_{l} \boldsymbol{\omega}_{i, l b}^{l b}\right) \mathbf{s}_{l}-k_{l q} \mathbf{T}_{l e}^{\top} \mathbf{e}_{l q}-k_{l \omega} \mathbf{s}_{l}\right] .
$$

A radial unbounded, positive definite Lyapunov function candidate is defined as

$$
\tilde{V}_{l}=\frac{1}{2}\left(\mathbf{s}_{l}^{\top} \mathbf{J}_{l} \mathbf{s}_{l}+\mathbf{e}_{l q}^{\top} k_{l q} \mathbf{e}_{l q}\right)>0 \quad \forall \mathbf{s}_{l} \neq \mathbf{0}, \mathbf{e}_{l q} \neq \mathbf{0},
$$

and, by differentiation of (59) and insertion of (58), we obtain

$$
\begin{aligned}
\dot{\tilde{V}}_{l} & =-\mathbf{s}_{l}^{\top} k_{l \omega} \mathbf{s}_{l}-\mathbf{e}_{l q}^{\top} \mathbf{T}_{l e} \Gamma k_{l q} \mathbf{T}_{l e}^{\top} \mathbf{e}_{l q}+\mathbf{s}_{l}^{\top} \boldsymbol{\tau}_{l d}^{l b} \\
& =-\boldsymbol{\chi}_{2}^{\top} \mathbf{Q}_{l} \boldsymbol{\chi}_{2}+\mathbf{s}_{l}^{\top} \boldsymbol{\tau}_{l d}^{l b} \\
& \leq-q l m\left\|\boldsymbol{\chi}_{2}\right\|^{2}+\beta_{l}\left\|\boldsymbol{\chi}_{2}\right\|,
\end{aligned}
$$

where $\boldsymbol{\chi}_{2}=\left[\mathbf{e}_{l q}^{\top}, \mathbf{s}_{l}^{\top}\right]^{\top}, \mathbf{Q}_{l}=\operatorname{diag}\left\{\mathbf{T}_{l e} k_{l q} \boldsymbol{\Gamma}_{l} \mathbf{T}_{l e}^{\top}, k_{l \omega} \mathbf{I}\right\}$, and $q_{l m}>0$ is the smallest eigenvalue of $\mathbf{Q}_{l}$. Accordingly, $\dot{\tilde{V}}_{l}<$ 0 when $\left\|\chi_{2}\right\|>\beta_{l} / q_{l m}=\delta_{2}$, and, as $\beta_{l}$ increases, it can be counteracted by increasing the controller gains. Hence, the perturbed system is uniformly practically asymptotically stable (UPAS); see [35]. We cannot claim semiglobal results for the same reasons as we cannot claim global results. Nevertheless, we assume that both $\Delta_{1}$ and $\Delta_{2}$ can be chosen arbitrary large to make it easier to follow the line of the proof.

4.2. Control of Follower Spacecraft. A similar disturbance as in Section 4.1 is introduced which are bounded such that $\left\|\tau_{f d}^{f b}\right\| \leq \beta_{f}$, and we apply a synchronizing controller based on the Slotine and Li structure such as

$$
\begin{aligned}
\boldsymbol{\tau}_{f a}^{l b}= & \mathbf{J}_{f} \dot{\boldsymbol{\omega}}_{i, r}^{f b}-\mathbf{S}\left(\mathbf{J}_{f} \boldsymbol{\omega}_{i, f b}^{f b}\right) \boldsymbol{\omega}_{i, r}^{f b}-k_{f q}\left(\mathbf{T}_{e f}^{\top} \mathbf{e}_{f q}-\mathbf{T}_{l e}^{\top} \mathbf{e}_{l q}\right) \\
& -k_{f \omega}\left(\mathbf{s}_{f}-\mathbf{s}_{l}\right),
\end{aligned}
$$

where $\boldsymbol{\omega}_{i, r}^{f b}$ and $\mathbf{s}_{f}$ are defined similar to (56)-(57). By inserting (61) into (9), we obtain

$$
\dot{\mathbf{s}}_{f}=\tilde{f}\left(\chi_{1}\right)+\tilde{g}(\chi) \chi_{2},
$$

where $\boldsymbol{\chi}_{1}=\left[\mathbf{e}_{f q}^{\top}, \mathbf{s}_{f}^{\top}\right]^{\top}$ and $\boldsymbol{\chi}=\left[\boldsymbol{\chi}_{1}^{\top}, \boldsymbol{\chi}_{2}^{\top}\right]^{\top}$, and $\tilde{f}\left(\boldsymbol{\chi}_{1}\right)$ and $\tilde{g}(\boldsymbol{\chi})$ are on the same form as (58) and (45), respectively.

The rest of the proof consists in verifying the conditions of [32, Theorem 3.3].

Proof of Assumption 3.2 (see [32]). The function $\tilde{g}(\boldsymbol{\chi}) \chi_{2}$ is uniformly bounded both in time and in $\theta_{2}$ and vanishes with $\chi_{2}$; thus, for any $\theta_{1} \in \Theta_{1}$, we choose

$$
\begin{gathered}
G_{\theta_{1}}(\|\chi\|)=\frac{1}{2}\left[\left(\frac{1}{J_{x}^{2}}+\frac{1}{J_{y}^{2}}+\frac{1}{J_{z}^{2}}\right)\left(k_{f q}^{2}+4 k_{f \omega}^{2}\right)\right]^{1 / 2}, \\
\Psi_{\theta_{1}}\left(\left\|\chi_{2}\right\|\right)=\left\|\chi_{2}\right\|,
\end{gathered}
$$

thus, $G_{\theta_{1}}(\|\chi\|)$ is constant and $\Psi_{\theta_{1}}\left(\left\|\chi_{2}\right\|\right)$ is of class $\mathcal{K}_{\infty}$, and the assumption is fulfilled for all $\theta_{2} \in \Theta_{2}$ and all $\chi \in 8^{3} \times$ $\mathbb{R}^{3} \times 8^{3} \times \mathbb{R}^{3}$.

Proof of Assumption 3.4 (see [32]). This Assumption was proven in Section 4.1.

Proof of Assumption 3.5 (see [32]). By applying the same reasoning as in Section 4.1, we achieve $\dot{\tilde{V}}_{f}<0$ when $\left\|\chi_{1}\right\|>\beta_{f} / q_{f m}=\delta_{1}$, where $q_{f m}>0$ is the smallest eigenvalue of $\mathbf{Q}_{f}=\operatorname{diag}\left\{\mathbf{T}_{f e} k_{f q} \boldsymbol{\Gamma}_{f} \mathbf{T}_{f e}^{\top}, k_{f \omega} \mathbf{I}\right\}$, which is defined similar to $\mathbf{Q}_{l}$ in (60). An increase of $\beta_{f}$ can as well be counteracted by increasing the controller gains; thus, given any $\Delta_{1}>\delta_{1}>0$, there exists a parameter $\theta_{1}^{\star}\left(\delta_{1}, \Delta_{1}\right) \in$ $\Theta_{1}$. We choose $\underline{\alpha}_{\delta_{1}, \Delta_{1}}\left(\left\|\chi_{1}\right\|\right)=1 / 2 \min \left\{j_{f m}, k_{f q}\right\}\left\|\chi_{1}\right\|^{2}$ and $\bar{\alpha}_{\delta_{1}, \Delta_{1}}\left(\left\|\chi_{1}\right\|\right)=1 / 2 \max \left\{j_{f M}, k_{f q}\right\}\left\|\chi_{1}\right\|^{2}$, and thus the first part of the assumption is fulfilled, and the second part is fulfilled for $\alpha_{\delta_{1}, \Delta_{1}}\left(\left\|\chi_{1}\right\|\right)=\min \left\{k_{f q} / 4, k_{f \omega}\right\}\left\|\chi_{1}\right\|^{2}$. The last inequality also holds similar to (51)-(53) with $c_{\delta_{1}, \Delta_{1}}\left(\left\|\chi_{1}\right\|\right)=$ $\sqrt{2} \sigma\left\|\chi_{1}\right\|^{1 / 2}$, and thus Assumption 3.5 holds for all $\chi_{1} \in$ $\mathcal{H}\left(\delta_{1}, \Delta_{1}\right)$, where $\Delta_{1}$ can be chosen arbitrary large by assumption.

Proof of Assumption 3.6 (see [32]). We define a LFC for the leader and follower spacecraft as

$$
\tilde{V}_{l f}(t, x)=\frac{1}{2}\left(\mathbf{s}_{l}^{\top} \mathbf{J}_{l} \mathbf{s}_{l}+\mathbf{e}_{l q}^{\top} k_{l q} \mathbf{e}_{l q}+\mathbf{s}_{f}^{\top} \mathbf{J}_{f} \mathbf{s}_{f}+\mathbf{e}_{f q}^{\top} k_{f q} \mathbf{e}_{f q}\right),
$$

which is lower and upper bounded by

$$
\begin{aligned}
& \underline{\alpha}_{l f}=\frac{1}{2} \min \left\{j_{l m}, j_{f m}, k_{l q}, k_{f q}\right\}\|\chi\|^{2}, \\
& \bar{\alpha}_{l f}=\frac{1}{2} \max \left\{j_{l M}, j_{f M}, k_{l q}, k_{f q}\right\}\|\chi\|^{2},
\end{aligned}
$$

respectively, and it can be shown that the second equation of [32, Proposition 2.13] is fulfilled by differentiation of (64). There exists a positive constant $\Delta_{0}$ such that for any given positive number $\delta_{1}, \Delta_{1}, \delta_{2}, \Delta_{2}$, satisfying $\Delta_{1}>\max \left\{\delta_{1}, \Delta_{0}\right\}$ 

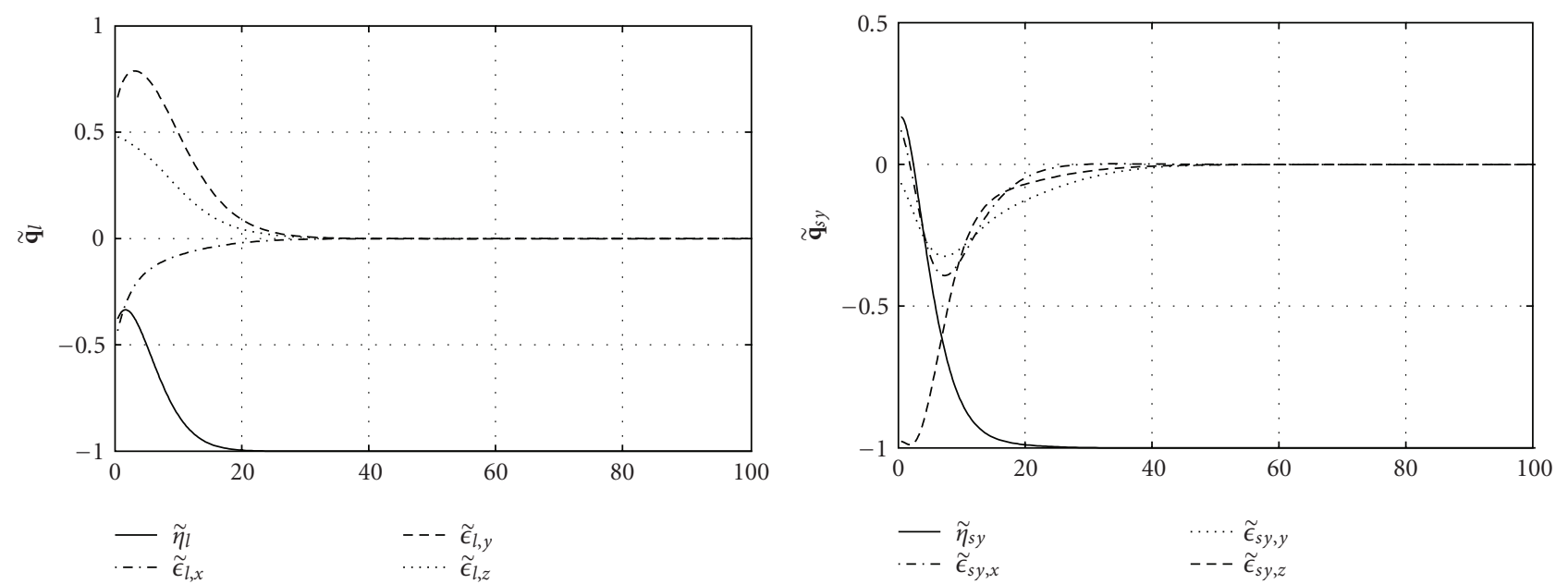

(a)
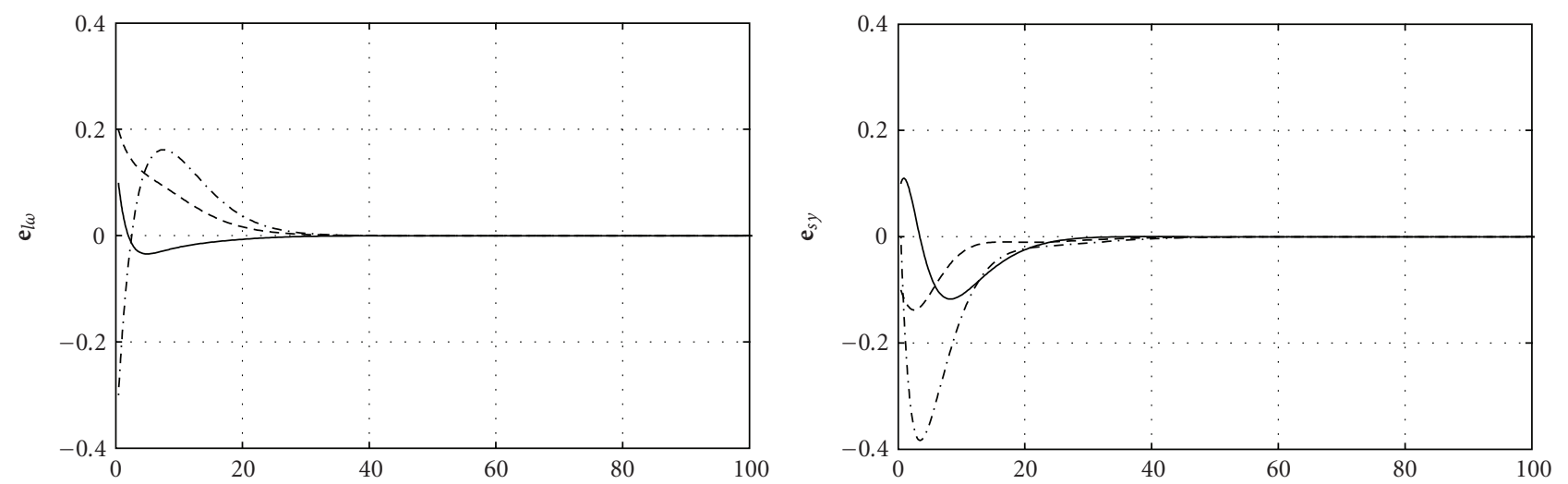

$$
\begin{array}{r}
-\mathbf{e}_{l, r} \\
-\cdot-\mathbf{e}_{l, \theta} \\
---\mathbf{e}_{l, h}
\end{array}
$$$$
\begin{aligned}
& -\mathbf{e}_{l, r} \\
& -\cdot-\mathbf{e}_{l, \theta} \\
& ---\mathbf{e}_{l, h}
\end{aligned}
$$

(c)
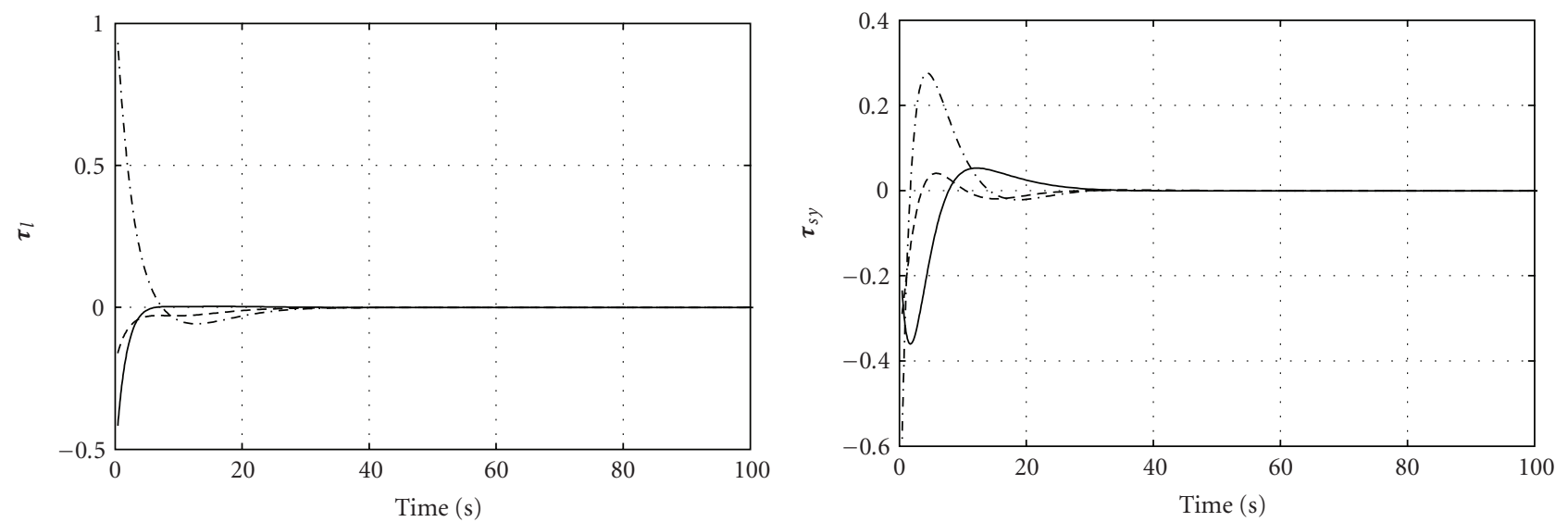

$$
\text { - } \boldsymbol{\tau}_{l, x}
$$$$
\begin{aligned}
& -\boldsymbol{\tau}_{s y, x} \\
& --\boldsymbol{\tau}_{s y, y} \\
& ---\tau_{s y, z}
\end{aligned}
$$

(e)

(f)

FIGURE 2: Attitude and angular velocity error and control torque for leader ((a), (c), (e)) and synchronizing follower ((b), (d), (f)) spacecraft during maneuver without measurement noise and disturbances. 


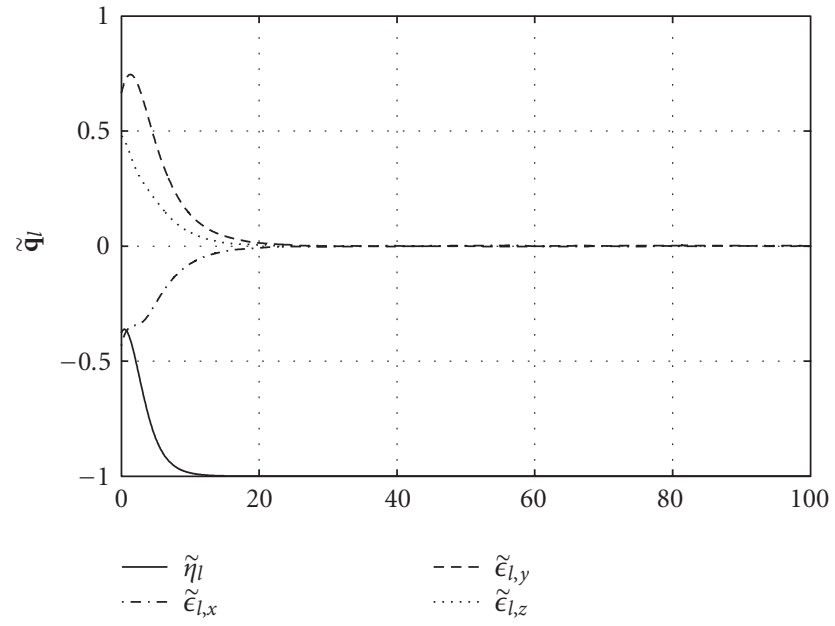

(a)

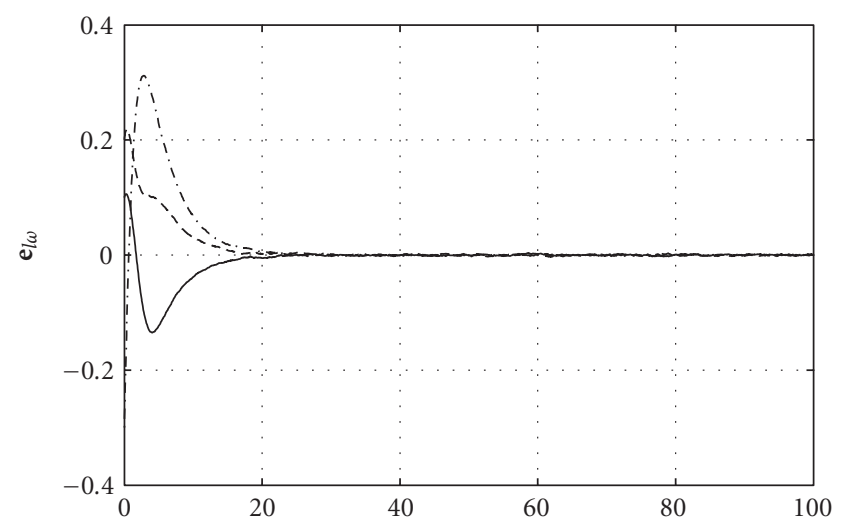

$$
\begin{array}{r}
-\mathbf{e}_{l, r} \\
-\cdot-\mathbf{e}_{l, \theta} \\
---\mathbf{e}_{l, h}
\end{array}
$$

(c)

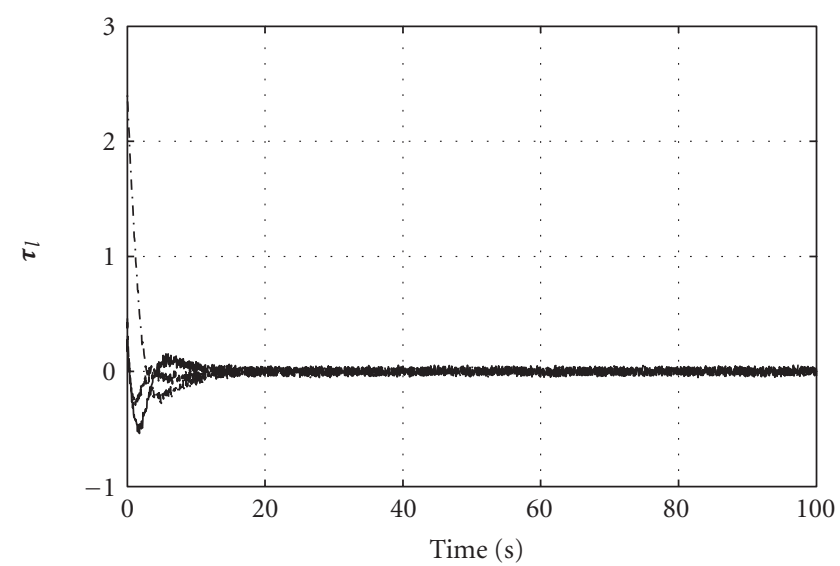

$$
\begin{array}{ll}
- & \tau_{l, x} \\
--- & \tau_{l, y} \\
--- & \tau_{l, z}
\end{array}
$$

(e)

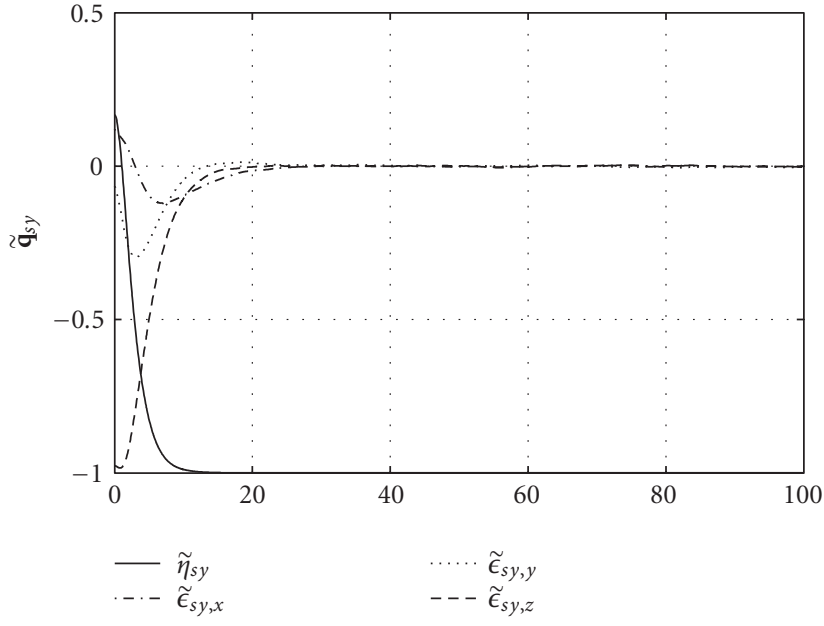

(b)

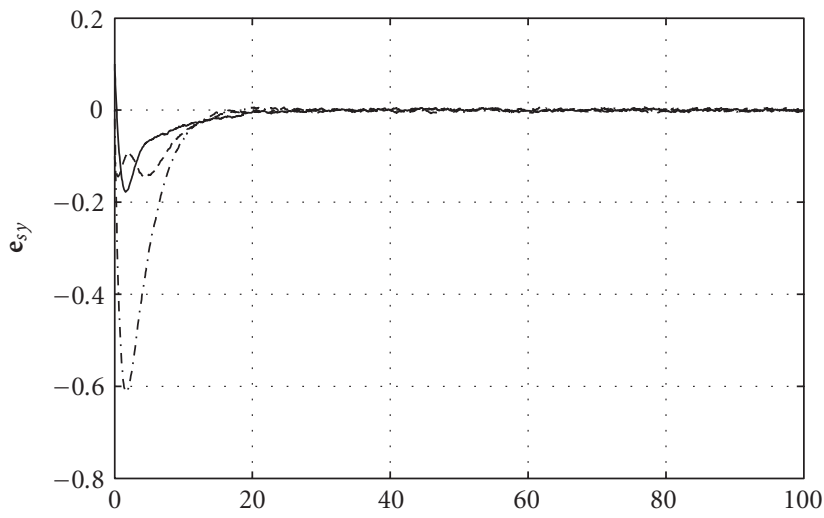

$$
\begin{array}{r}
-\mathbf{e}_{l, r} \\
-\cdot-\mathbf{e}_{l, \theta} \\
---\mathbf{e}_{l, h}
\end{array}
$$

(d)

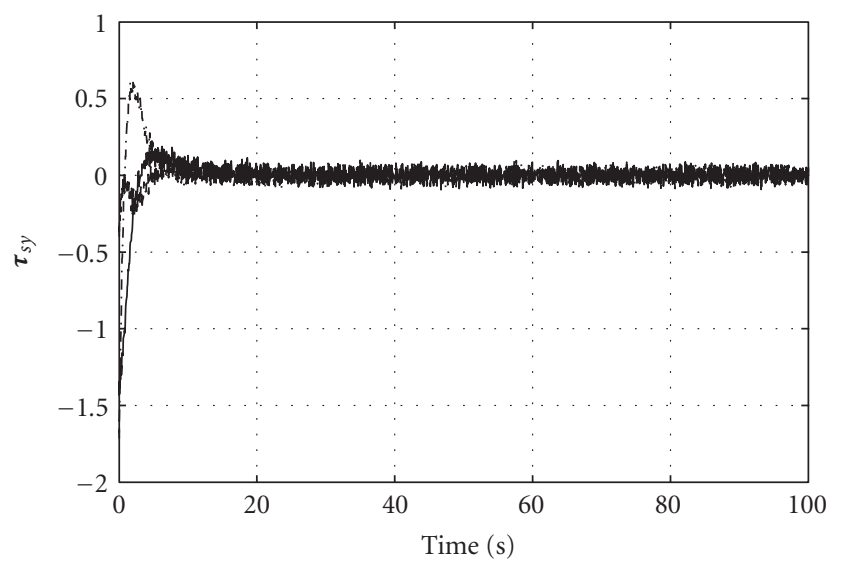

$$
\begin{array}{ll}
- & \boldsymbol{\tau}_{s y, x} \\
-.- & \boldsymbol{\tau}_{s y, y} \\
--- & \boldsymbol{\tau}_{s y, z}
\end{array}
$$

(f)

Figure 3: Attitude and angular velocity error and control torque for leader ((a, (c), (e))) and synchronizing follower ((b, (d), (f))) spacecraft during maneuver including measurement noise and disturbances. 
and $\Delta_{2}>\delta_{2}$, there exists a $\delta_{1}$ such that $\bar{\alpha}_{l f}\left(\delta_{1}\right)<\underline{\alpha}_{l f}\left(\Delta_{1}\right)$. As mentioned in Section 4.1, given any $\beta_{l}$, we can achieve any $\delta_{2}$ by increasing the gains $\theta_{2}$, and thus there exits a parameter $\theta_{2}^{\star} \in \mathcal{D}_{f_{2}}\left(\delta_{2}, \Delta_{2}\right) \cap \Theta_{2}$, such that by applying the last inequality of [32, Proposition 2.13] using the bounds (65), we see that the first equation of Assumption 3.6 is fulfilled for

$$
\gamma\left(\Delta_{1}\right)=\sqrt{\frac{\min \left\{j_{l m}, j_{f m}, k_{l q}, k_{f q}\right\} \Delta_{1}^{2}}{\max \left\{j_{l M}, j_{f M}, k_{l q}, k_{f q}\right\}} .}
$$

We have that

$$
\underline{\alpha}_{\delta_{1}, \Delta_{1}}^{-1} \circ \bar{\alpha}_{\delta_{1}, \Delta_{1}}\left(\delta_{1}\right)=\sqrt{\frac{\max \left\{j_{f M}, k_{f q}\right\} \delta_{1}^{2}}{\min \left\{j_{f m}, k_{f q}\right\}}},
$$

then, for any $\Delta_{\star}>\delta_{\star}>0$, there exist parameters $\delta_{1}, \delta_{2}, \Delta_{1}$, and $\Delta_{2}$ such that

$$
\min \left\{\Delta_{1}, \Delta_{2}, \sqrt{\frac{\min \left\{j_{l m}, j_{f m}, k_{l q}, k_{f q}\right\} \Delta_{1}^{2}}{\max \left\{j_{l M}, j_{f M}, k_{l q}, k_{f q}\right\}}}\right\} \geq \Delta_{\star}
$$

since $\Delta_{1}$ and $\Delta_{2}$ can be chosen arbitrarily large and the constants $j_{s m}, j_{s M}, k_{s q}, k_{s \omega}>0$, and

$$
\max \left\{\delta_{2}, \sqrt{\frac{\max \left\{j_{f M}, k_{f q}\right\} \delta_{1}^{2}}{\min \left\{j_{f m}, k_{f q}\right\}}}\right\} \leq \delta_{\star}
$$

is fulfilled since, by decreasing $\delta_{1}, k_{f q}$ is increased but only of order one, and thus the two last inequalities of Assumption 3.6 are fulfilled and Assumption 3.6 holds. It can then be concluded that the equilibrium points of the cascaded system are UPAS.

By setting $\mathbf{e}_{l q}=\mathbf{e}_{l q_{-}}$or $\mathbf{e}_{f q}=\mathbf{e}_{f q_{-}}$, the other three proofs are performed in a similar way, and we thus conclude that the set of equilibrium points $\left(\mathbf{e}_{l q \pm}, \mathbf{e}_{l \omega}, \mathbf{e}_{f q_{ \pm}}, \mathbf{e}_{f \omega}\right)=(\mathbf{0}, \mathbf{0}, \mathbf{0}, \mathbf{0})$ are UPAS.

\section{Simulations}

In the following, simulation results of a leader spacecraft in an elliptic LEO with the follower spacecraft following the same orbit with one-second delay are presented to validate the proposed approach. The simulations were performed in Simulink using a variable sample-time Runge-Kutta ODE45 solver with relative and absolute tolerance of $10^{-9}$. The moments of inertia were chosen as $\mathbf{J}_{l}=\mathbf{J}_{f}=$ $\operatorname{diag}\{4.35,4.33,3.664\} \mathrm{kgm}^{2}$, and the spacecraft orbits were chosen with apogee at $750 \mathrm{~km}$, perigee at $600 \mathrm{~km}$, inclination at $71^{\circ}$, and the argument of perigee and the right ascension of the ascending node at $0^{\circ}$. The initial conditions were chosen as $\mathbf{q}_{l}\left(t_{0}\right)=[-0.3772,-0.4329,0.6645,0.4783]^{\top}, \mathbf{q}_{f}\left(t_{0}\right)=$ $1 / \sqrt{4}[1,1,1,1]^{\top}, \boldsymbol{\omega}_{l}\left(t_{0}\right)=[0.1-0.30 .2]^{\top}$, and $\boldsymbol{\omega}_{f}\left(t_{0}\right)=$ $[0.2-0.30 .1]^{\top}$, controller gains as $k_{s q}=1, k_{s \omega}=2$, and $\Gamma_{s}=$ I for (23), (43), (55), and (61), and desired trajectory as $\dot{\boldsymbol{\omega}}_{d}=0.1\left[-10 c_{0}^{2} \cos \left(8 c_{0} t\right), 48 c_{0}^{2} \sin \left(16 c_{0} t\right),-8 c_{0}^{2} \cos \left(4 c_{0} t\right)\right]$, $\boldsymbol{\omega}_{d}$ its integrate, and $\mathbf{q}_{d}\left(t_{0}\right)=[1, \mathbf{0}]^{\top}$, where $c_{0}=\pi / t_{o}$ and $t_{o}$ denotes the orbital period. For relative attitude (synchronization error) and angular velocity between the leader and follower spacecraft, we define $\tilde{\mathbf{q}}_{s y}=\mathbf{q}_{f} \otimes \overline{\mathbf{q}}_{l}$ and $\mathbf{e}_{s y}=\mathbf{e}_{f \omega}-\mathbf{e}_{l \omega}$, respectively.

In Figure 2, simulation results are presented without disturbances and noise. From Figure 2(a) we see that the leader spacecraft settles at the negative equilibrium, the angular velocity error go towards zero, and the actuator torque is presented in the bottommost plot. On the right-hand side we see that the relative attitude and angular velocity error go towards zero, and thus the follower spacecraft is able to synchronize with the leader spacecraft. The bottommost plot on the right hand depicts the follower actuator torque.

In the second set of simulation results, we introduce measurement noise as $\sigma \mathbb{B}^{n}=\left\{x \in \mathbb{R}^{n}:\|x\| \leq \sigma\right\}$ which was added according to $\widetilde{\mathbf{e}}_{q}=\left(\mathbf{e}_{q}+0.05 \mathbb{B}^{4}\right) /\left\|\mathbf{e}_{q}+0.05 \mathbb{B}^{4}\right\|$ and $\widetilde{\mathbf{e}}_{\omega}=\mathbf{e}_{\omega}+0.01 \mathbb{B}^{3}$ for both the leader and follower spacecraft. Also, since we are considering a slightly elliptic LEO, we only considered the disturbance torques which are the major contributors to these kind of orbits, that is, gravity gradient torque and torques caused by atmospheric drag and $J_{2}$ effect (cf. $[34,36,37]$ ). The latter is caused by nonhomogeneous mass distribution of a planet. The torques generated by atmospheric drag and $J_{2}$ were induced because of a $10 \mathrm{~cm}$ displacement of the center of mass. All disturbances are considered continuous and bounded.

As it can be seen from Figure 3, the control laws derived for unknown disturbances also are able to make the leader track the reference and make the follower synchronize with the leader, similar to the results from the first simulation. One notable difference is that these control laws are in general faster than the results presented in Figure 2, though demanding larger absolute control torque.

\section{Conclusions}

In this paper, we have presented control laws for leaderfollower synchronization of spacecraft, performed stability analysis based on cascade theory, and proven the equilibrium points of the overall system to be uniformly asymptotically stable (UAS) when disturbances were assumed to be known, and uniformly practically asymptotically stable (UPAS) when unknown, but bounded disturbances were included. Simulation results were presented to validate the proposed method for the overall system showing that the follower spacecraft was able to synchronize with the leader spacecraft in a satisfactor way even when disturbances were presented.

\section{References}

[1] I. Blekhman, Synchronization in Science and Technology, ASME Press Translations, ASME Press, New York, NY, USA, 1988.

[2] A. Rodriguez-Angeles, Synchronization of mechanical systems, Ph.D. thesis, Eindhoven University of Technology, Eindhoven, The Netherlands, 2002.

[3] H. Nijmeijer and A. Rodriguez-Angeles, Synchronization of Mechanical Systems, vol. 46 of World Scientific Series on Nonlinear Science, Series A, 2003. 
[4] E. Kyrkjebø, Motion coordination of mechanical systems: leaderfollower synchronization of euler-lagrange systems using output feedback control, Ph.D. thesis, Department of Engineering Cybernetics, Norwegian University of Science and Technology, Trondheim, Norway, 2007.

[5] A. Rodriguez-Angeles and H. Nijmeijer, "Coordination of two robot manipulators based on position measurements only," International Journal of Control, vol. 74, no. 13, pp. 1311-1323, 2001.

[6] A. K. Bondhus, K. Y. Pettersen, and H. Nijmeijer, "Masterslave synchronization of robot manipulators," in Proceedings of the IFAC Symposium on Nonlinear Control Systems Design, Stuttgart, Germany, 2004.

[7] J. Lawton and R. W. Beard, "Elementary attitude formation maneuver via leader-following and behaviour-based control," in Proceedings of the AIAA Guidance, Navigation and Control Conference, Denver, Colo, USA, 2000.

[8] A. K. Bondhus, K. Y. Pettersen, and J. T. Gravdahl, "Leader/follower synchronization of satellite attitude without angular velocity measurements," in Proceedings of the 44th IEEE Conference on Decision and Control, and the European Control Conference (CDC-ECC '05), pp. 7270-7277, Seville, Spain, December 2005.

[9] R. Kristiansen, Dynamic synchronization of spacecraftmodeling and coordinated control of leader-follower spacecraft formations, Ph.D. thesis, Department of Engineering Cybernetics, Norwegian University of Science and Technology, Trondheim, Norway, 2008.

[10] E. I. Grøtli, Robust stability and control of spacecraft formations, Ph.D. thesis, Norwegian University of Science and Technology, 2010.

[11] S. H. Fu, C. C. Cheng, and C. Y. Yin, "Nonlinear adaptive tracking control for underway replenishment process," in Proceeding of IEEE International Conference on Networking, Sensing and Control, vol. 2, pp. 707-712, Taipei, Taiwan, March 2004.

[12] E. Kyrkjebø, E. Panteley, A. Chaillet, and K. Y. Pettersen, "A virtual vehicle approach to underway replenishment," in Group Coordination and Cooperative Control, K. Y. Pettersen, J. T. Gravdahl, and H. Nijmeijer, Eds., vol. 336 of Lecture Notes in Control and Information Sciences, pp. 171-189, Springer, Berlin, Germany, 2006.

[13] J. R. Lawton, B. J. Young, and R. W. Beard, "Decentralized approach to elementary formation maneuvers," in Proceedings of IEEE International Conference on Robotics and Automation (ICRA '00), vol. 3, pp. 2728-2733, April 2000.

[14] G. Antonelli and S. Chiaverini, "Kinematic control of platoons of autonomous vehicles," IEEE Transactions on Robotics, vol. 22, no. 6, pp. 1285-1292, 2006.

[15] F. Arrichiello, Coordination control of multiple mobile robots, Ph.D. thesis, Cassino University, Cassino, Italy, 2006.

[16] F. Arrichiello, S. Chiaverini, and T. I. Fossen, "Formation control of marine surface vessels using the null-space-based behavioral control," in Group Coordination and Cooperative Control, K. Y. Pettersen, J. T. Gravdahl, and H. Nijmeijer, Eds., vol. 336 of Lecture Notes in Control and Information Sciences, chapter 1, pp. 1-19, Springer, Berlin, Germany, 2006.

[17] R. Cui, S. S. Ge, B. voon Ee How, and Y. Sang Choo, "Leaderfollower formation control of underactuated autonomous underwater vehicles," Ocean Engineering, vol. 37, no. 17-18, pp. 1491-1502, 2010.

[18] H. Bai, M. Arcak, and J. T. Wen, "Adaptive motion coordination: using relative velocity feedback to track a reference velocity," Automatica, vol. 45, no. 4, pp. 1020-1025, 2009.
[19] A. Sarlette, R. Sepulchre, and N. E. Leonard, "Autonomous rigid body attitude synchronization," Automatica, vol. 45, no. 2, pp. 572-577, 2009.

[20] D. V. Dimarogonas, P. Tsiotras, and K. J. Kyriakopoulos, "Leader-follower cooperative attitude control of multiple rigid bodies," Systems and Control Letters, vol. 58, no. 6, pp. 429435, 2009.

[21] R. Ortega, A. Loría, P. J. Nicklasson, and H. Sira-Ramírez, Passivity-based Control of Euler-Lagrange Systems: Mechanical, Electrical and Electromechanical Applications, Comunications and Control Engineering, Springer, London, UK, 1998.

[22] B. Paden and R. Panja, "Globally asymptotically stable 'PD+' controller for robot manipulators," International Journal of Control, vol. 47, no. 6, pp. 1697-1712, 1988.

[23] J. J. E. Slotine and W. Li, "On the adaptive control of robot manipulators," International Journal of Robotics Research, vol. 6, no. 3, pp. 49-59, 1987.

[24] F. Mazenc, L. Praly, and W. P. Dayawansa, "Global stabilization by output feedback: examples and counterexamples," Systems and Control Letters, vol. 23, no. 2, pp. 119-125, 1994.

[25] A. Loría and E. Panteley, "Cascaded nonliear time-varying systems: analysis and design," in Advanced Topics in Control Systems Theory, vol. 311 of Lecture Notes in Control and Information Sciences, chapter 2, pp. 23-64, Springer, Berlin, Germany, 2005.

[26] T. I. Fossen and O.-E. Fjellstad, "Cascaded adaptive control of ocean vehicles with significant actuator dynamics," in Proceedings of the IFAC World Congress, Sydney, Australia, 1993.

[27] A. Loría, H. Nijmeijer, and O. Egeland, "Cascaded synchronization of two pendula," in Proceedings of the American Control Conference, 1998.

[28] M. Jankovic, R. Sepulchre, and P. V. Kokotovic, "Constructive Lyapunov stabilization of nonlinear cascade systems," IEEE Transactions on Automatic Control, vol. 41, no. 12, pp. 17231735, 1996.

[29] Z. P. Jiang and I. M. Y. Mareels, "A small-gain control method for nonlinear cascaded systems with dynamic uncertainties," IEEE Transactions on Automatic Control, vol. 42, no. 3, pp. 292-308, 1997.

[30] E. Panteley and A. Loria, "On global uniform asymptotic stability of nonlinear time-varying systems in cascade," Systems and Control Letters, vol. 33, no. 2, pp. 131-138, 1998.

[31] A. Chaillet and A. Loría, "Uniform semiglobal practical asymptotic stability for non-autonomous cascaded systems and applications," Automatica, vol. 44, no. 2, pp. 337-347, 2008.

[32] A. Chaillet, On stability and robustness of nonlinear systems: applications to cascaded systems, Ph.D. thesis, UFR Scientifique D’Orsay, Paris, France, 2006.

[33] O. Egeland and J. T. Gravdahl, Modeling and Simulation for Automatic Control, Marine Cybernetics, Trondheim, Norway, 2002.

[34] M. J. Sidi, Spacecraft Dynamics and Control, Cambridge University Press, New York, NY, USA, 1997.

[35] W. Hahn, Stability of Motion, Springer, Berlin, Germany, 1967.

[36] O. Montenbruck and E. Gill, Satellite Orbits. Models, Methods, Applications, Springer, Berlin, Germany, 1st edition, 2001.

[37] J. R. Wertz, Ed., Spacecraft Attitude Determination and Control, Kluwer Academic Publishers, London, UK, 1978.

[38] Ø. Hegrenæs, J. T. Gravdahl, and P. Tøndel, "Spacecraft attitude control using explicit model predictive control," Automatica, vol. 41, no. 12, pp. 2107-2114, 2005. 
[39] T. I. Fossen, Marine Control Systems: Guidance, Navigation, and Control of Ships, Rigs and Underwater Vehicles, Marine Cybernetics, Trondheim, Norway, 2002.

[40] J. T. Y. Wen and K. Kreutz-Delgado, "The attitude control problem," IEEE Transactions on Automatic Control, vol. 36, no. 10, pp. 1148-1162, 1991.

[41] A. Loría, E. Panteley, D. Popovic, and A. R. Teel, " $\delta$-persistency of excitation: a necessary and sufficient condition for uniform attractivity," in Proceedings of the 41st IEEE Conference on Decision and Control, vol. 3, pp. 3506-3511, Las Vegas, Nev, USA, December 2002. 

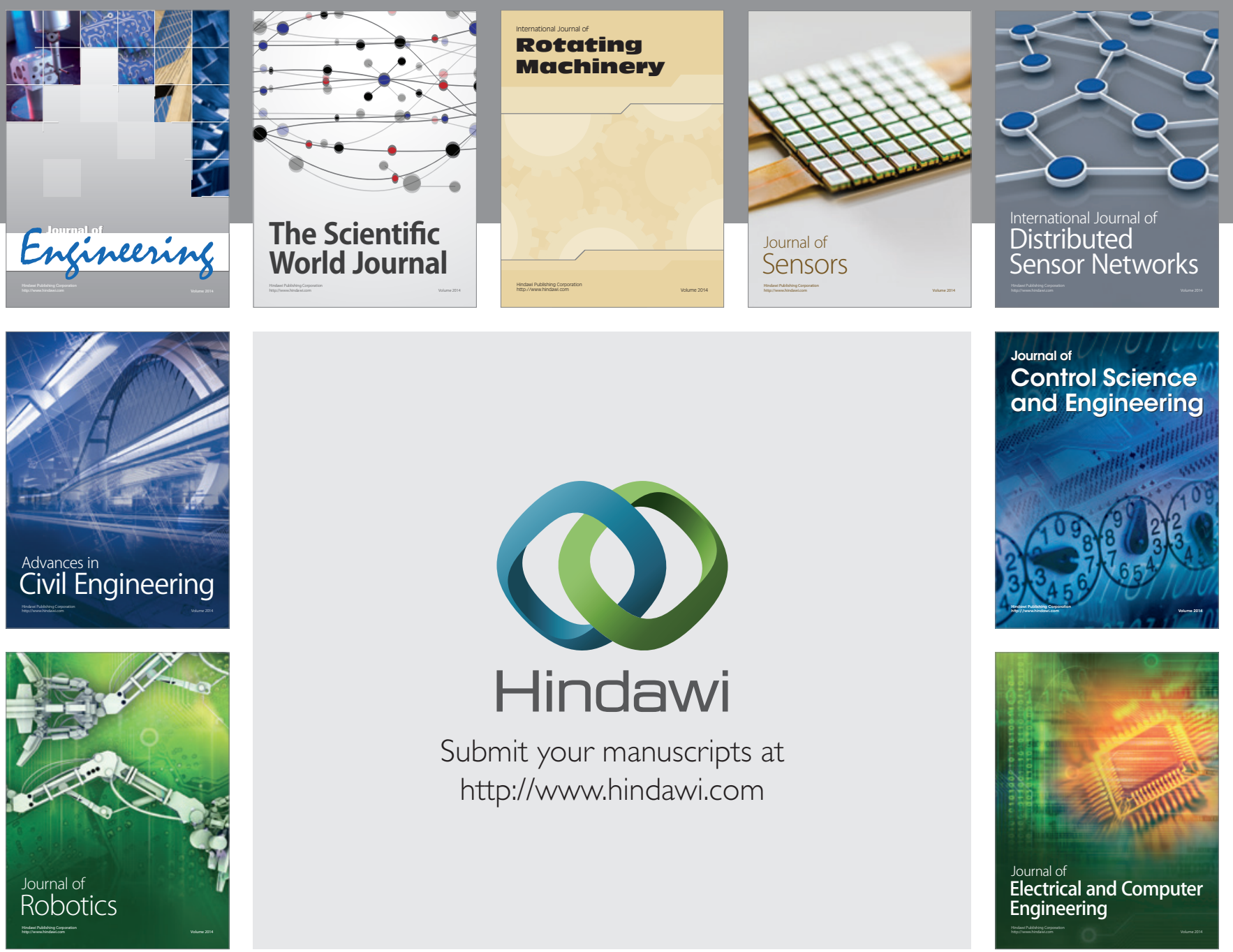

Submit your manuscripts at

http://www.hindawi.com
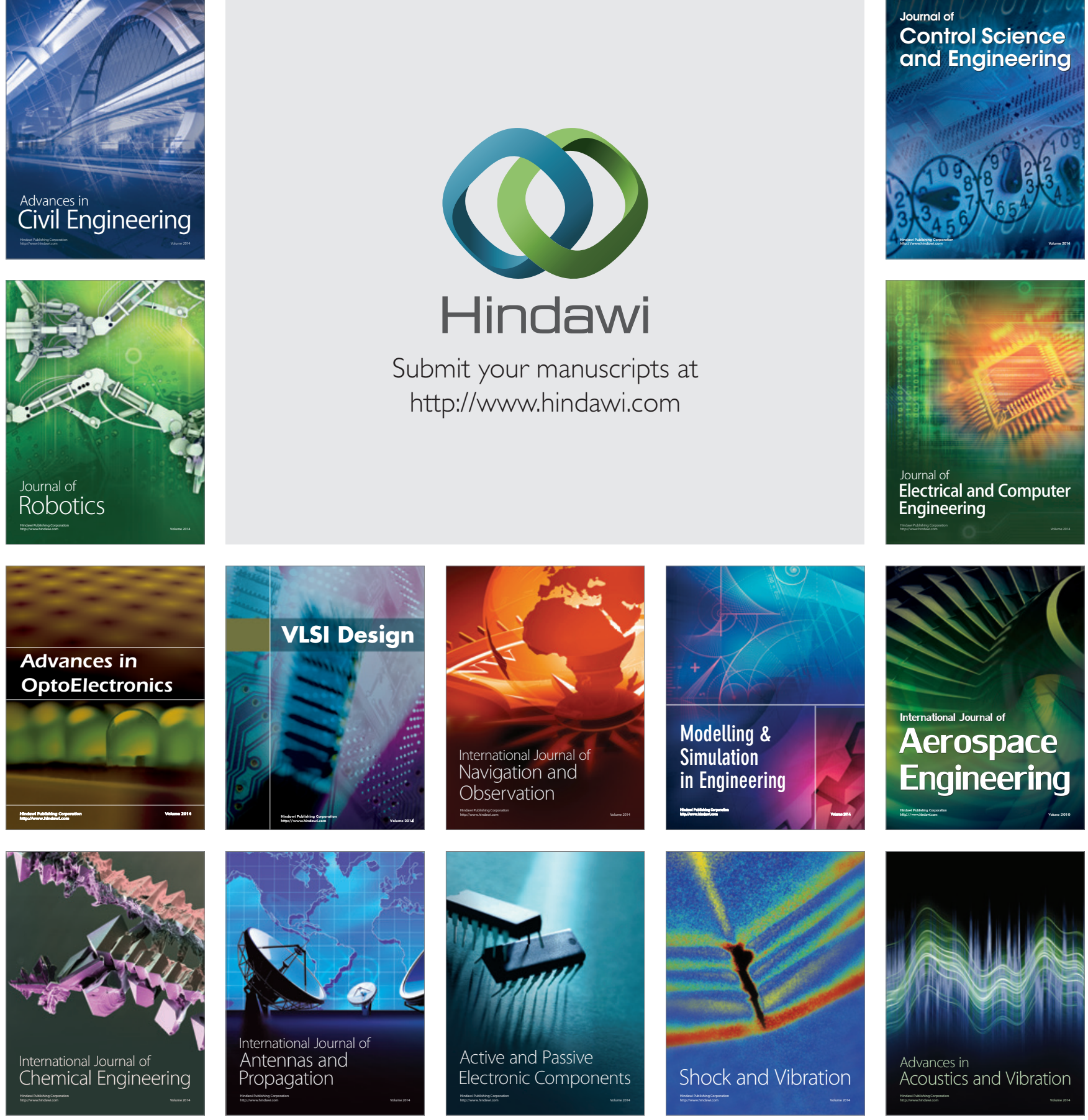